NASA/CR-1998-208701

ICASE Report No. 98-38

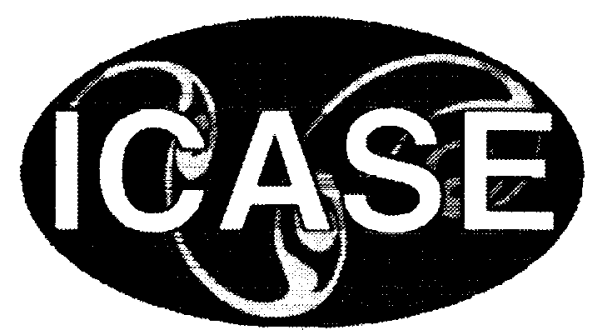

\title{
Complete Galilean-Invariant Lattice BGK Models for the Navier-Stokes Equation
}

Yue-Hong Qian

Columbia University, New York, New York

Ye Zhou

ICASE, Hampton, Virginia

and

IBM, Yorktown Heights, New York

Institute for Computer Applications in Science and Engineering NASA Langley Research Center

Hampton, VA

Operated by Universities Space Research Association

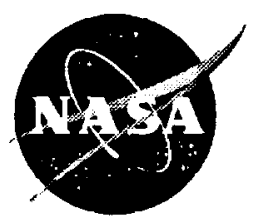

National Aeronautics and

Space Administration

Langley Research Center

Hampton, Virginia 23681-2199

Prepared for Langley Research Center under Contract NAS1-97046 
Available from the following:

NASA Center for AeroSpace Information (CASI)

7121 Standard Drive

Hanover, MD 21076-1320

(301) 621-0390
National Technical Information Service (NTIS)

5285 Port Royal Road

Springfield, VA 22161-2171

(703) $487-4650$ 


\title{
COMPLETE GALILEAN-INVARIANT LATTICE BGK MODELS FOR THE NAVIER-STOKES EQUATION
}

\author{
YUE-HONG QIAN* AND YE ZHOU ${ }^{\dagger}$
}

\begin{abstract}
Galilean invariance has been an important issue in lattice-based hydrodynamics models. Previous models concentrated on the nonlinear advection term. In this paper, we take into account the nonlinear response effect in a systematic way. Using the Chapman-Enskog expansion up to second order, complete Galilean invariant lattice BGK models in one dimension $(\theta=3)$ and two dimensions $(\theta=1)$ for the Navier-Stokes equation have been obtained.
\end{abstract}

Key words. Boltzmann equation, lattice-based hydrodynamics models, Navier-Stokes equation, Galilean invariance

Subject classification. Fluid Mechanics

1. Introduction. The modern version of Lattice Gas Automata (LGA) hydrodynamics model, introduced by Frisch, Hasslacher and Pomeau [1-2], has attracted much attention since 1986. In particular, the natural advantage of lattice-based models for performing parallel computation and handling complex geometries problems, with local operations, indicates the lattice models may provide an alternative efficient numerical scheme for studying many complex phenomena [3-8].

However, the disadvantages of LGA models are rather significant. Boolean operations are intrinsically noisy. The pressure also contains an explicit velocity dependence, which is obviously unphysical. Furthermore, LGA could not satisfy Galilean invariant due to the density dependence of a so-called $g(\rho)$ factor [9]. We stress that Galilean invariance is a basic requirement that is demanded for any physical model.

Steps were taken to overcome the difficulties of LGA. McNamara and Zanetti [10] replaced the Boolean operations by neglecting particle correlations and introducing averaged distribution functions. Therefore, the computational accuracy is improved by eliminating the statistical noise. Higuera and Jimenez [11] noted that there are many choices for the collision integral that could generate the same equilibrium distribution. In 1992, the Bhatnagar-Gross-Krook approximation for lattice Boltzmann was independently introduced by Qian et al. [12-13] and Chen et al. [14-15]. The key observations in these two studies are that the requirement for using Fermi-Dirac statistics is no longer necessary and that of the resulted freedom in the functional form of the equilibrium distribution provide many choices for using desired equilibrium distribution functions. The Galilean invariance can be easily achieved for advection terms.

Qian and Orszag [16] found a cubic nonlinear deviation from the Navier-Stoke equations when nonlinear response was taken into account. The cubic nonlinear term is important in compressible regime when the Mach number is not small, as usually assumed in the LGA models. Note that the cubic term, as a direct result of popular equilibrium functions, is hidden in all lattice Boltzmann calculations. Note that Chen et al. [17] attempted to eliminate the cubic nonlinear deviation term. Their effort was motivated by the fact

\footnotetext{
*Department of Applied Physics and Applied Mathematics, Columbia University, New York, NY 10027.

† Institute for Computer Applications in Science and Engineering, NASA Langley Research Center, Hampton, VA 23681 and IBM Research Division, T.J. Watson Research Center, P.O. Box, 218, Yorktown Heights, NY 10598. This research was supported by the National Aeronautics and Space Administration under NASA Contract No. NAS1-97046 while the second author was in residence at the Institute for Computer Applications in Science and Engineering (ICASE), NASA Langley Research Center, Hampton, VA 23681.
} 
that the cubic terms is not part of original Navier-Stokes equation. They used an artificial procedure by introducing and manipulating higher order tensors of the expanded equilibrium distribution function in a rather complicated manner.

The purpose of this letter is to point out that current lattice Boltzmann and lattice BGK models have not achieved a complete Galilean invariance. Indeed, we must emphase here that the cubic nonlinear deviation term is not Galilean invariant when $\mathbf{u} \rightarrow \mathbf{u}+\mathbf{U}_{\mathbf{0}}$ where $\mathbf{U}_{\mathbf{0}}$ is a constant velocity. We will present an expanded discussion on the Galilean invariance issue in next section and develop a 5-velocity model in one dimension $(\theta=3.0)$ and a 17-velocity model in two dimensions $(\theta=1.0)$ to eliminate the cubic nonlinear term. As a result, these two models achieve a status of complete Galilean invariance up to second order derivative since no higher-order dynamics exists [18]. Along the way, these two models remove another shortcoming of the lattice Boltzmann schemes, the staggered spurious invariants, as pointed out by the paper [19].

2. Galilean Invariance and Models Description. The original FHP model [1] has several weaknesses. Non-Galilean invariance is one of such problems. Much effort has been made to get the Galilean invariance in the nonlinear advection term, first by lattice gas models [9], then by lattice BGK models [1215]. Qian [12] pointed out that the problem of non-Galilean invariance is directly related to the problem of velocity-dependent pressure, the two problems became one. When deriving large-scale dynamics, the models use linear response of small perturbation. If nonlinear response effect is taken into account, a term deviating from the Navier-Stokes equation is obtained [16]. This new term is non-Galilean invariant and this fact hasn't been pointed out so far as we know. The consequence of this cubic nonlinear term leads to a frame-velocity dependent viscosity, which is not physical. We shall recall first the previous models before introducing a tunable parameter $\theta$ to control the cubic nonlinear term.

Time evolution of lattice BGK models is the following [13],

$$
f_{i}\left(\vec{x}+\vec{c}_{i}, t+1\right)=f_{i}(\vec{x}, t)+\omega\left[f_{i}^{e}(\vec{x}, t)-f_{i}(\vec{x}, t)\right]
$$

where $f_{i}$ is the particle density with given velocity $\overrightarrow{c_{i}}$ and $\omega$ the relaxation parameter $(0 \leq \omega \leq 2) . i$ is the index of discrete velocity $\overrightarrow{c_{i}}$. A workable equilibrium $f_{i}^{e}$ leading to hydrodynamic equations is [12-13],

$$
f_{i}^{e}=\rho t_{p}\left[1+\frac{c_{i \alpha} u_{\alpha}}{c_{s}^{2}}+\frac{u_{\alpha} u_{\beta}}{2 c_{s}^{4}}\left(c_{i \alpha} c_{i \beta}-c_{s}^{2} \delta_{\alpha \beta}\right)\right]
$$

where $t_{p}$ is a lattice structure weighting factor (the index $p=c_{i}^{2}$ ) and $c_{s}$ is a constant. A slightly different form of equilibrium distribution for the FHP hexagonal lattice was used by Chen et al. [15]. The conditions to determine the values of $t_{p}$ are the constraints of conservation laws, the isotropic fourth order tensor of velocity and Galilean invariance in advection term. Greek subscripts $\alpha, \beta$ denote the space directions in Cartesian coordinates. The conventional summation of repeated Greek subscripts is used. The hydrodynamic density $\rho$ and velocity $\vec{u}$ are defined as,

$$
\rho=\sum_{i} f_{i}=\sum_{i} f_{i}^{e}, \quad \rho \vec{u}=\sum_{i} f_{i} \vec{c}_{i}=\sum_{i} f_{i}^{e} \vec{c}_{i}
$$

Using the Chapman-Enskog expansion, we derive the hydrodynamic equations up to the second order of Knudsen number at long wavelength and long time limits,

$$
\begin{aligned}
\partial_{t} \rho+\partial_{\alpha}\left(\rho u_{\alpha}\right)= & 0 \\
\partial_{t}\left(\rho u_{\alpha}\right)+\partial_{\beta}\left(\rho u_{\alpha} u_{\beta}\right)= & -\partial_{\alpha} P+\partial_{\beta}\left[\rho \nu \partial_{\beta}\left(u_{\alpha}\right)+\rho \zeta \partial_{\alpha}\left(u_{\beta}\right)\right] \\
& -\sigma \partial_{\beta} \partial_{\gamma}\left(\rho u_{\alpha} u_{\beta} u_{\gamma}\right)
\end{aligned}
$$


where $\nu$ and $\zeta$ are shear and bulk viscosities [13],

$$
\nu=\zeta=\frac{c_{s}^{2}}{2}\left(\frac{2}{\omega}-1\right)
$$

The equation of state $P(\rho)$ is,

$$
P=c_{s}^{2} \rho
$$

$\sigma$ is directly related to $\omega$ by,

$$
\sigma=\frac{1}{\omega}-\frac{1}{2}
$$

The $\sigma$ cubic nonlinear term is the new term in eq. (2.5) which has been largely ignored in the community [16], the physical meaning of this term is the nonlinear response due to the quadratic term in the equilibrium (2.2).

We argue that this term is also a source of non-Galilean invariance. With a uniformly moving frame velocity $U_{0}$, we have the following variable transformation: $t \rightarrow t, x \rightarrow x-U_{0} t, u \rightarrow u+U_{0}$ and $\rho \rightarrow \rho$. The momentum in the moving frame is now,

$$
\begin{aligned}
\partial_{t}\left(\rho u_{\alpha}\right)+\partial_{\beta}\left(\rho u_{\alpha} u_{\beta}\right)= & -\partial_{\alpha} P+\partial_{\beta}\left[\rho \nu \partial_{\beta}\left(u_{\alpha}\right)+\rho \zeta \partial_{\alpha}\left(u_{\beta}\right)\right] \\
& -\sigma \partial_{\beta} \partial_{\gamma}\left[\rho\left(u+U_{0}\right)_{\alpha}\left(u+U_{0}\right)_{\beta}\left(u+U_{0}\right)_{\gamma}\right]
\end{aligned}
$$

When measuring the shear viscosity, we linearize the above equations by assuming that $U_{0}$ is in $x$ direction,

$$
\partial_{t} v=\left(\nu-\sigma U_{0}^{2}\right) \partial_{x} \partial_{x} v
$$

where $v$ is the transverse small velocity. Therefore, the effective shear viscosity $\nu_{\text {eff }}$ is,

$$
\nu_{e f f}=\frac{c_{s}^{2}-U_{0}^{2}}{2}\left(\frac{2}{\omega}-1\right)
$$

The velocity-dependence of viscosity is also unphysical. We will verify this relation numerically.

We now use a modified equilibrium distribution for a one-dimensional 5-velocity model (D1Q5): $(0, \pm 1, \pm 2)$ and a two-dimensional 17-velocity model (D2Q17): $(0,0),( \pm 1,0),(0, \pm 1),( \pm 1, \pm 1),( \pm 2,0),(0, \pm 2)$ and $( \pm 2, \pm 2)$. We add a cubic nonlinear term to the equilibrium distribution with an ajustable parameter $\theta$

$$
f_{i}^{e}=\rho t_{p}\left[1+\frac{c_{i \alpha} u_{\alpha}}{c_{s}^{2}}+\frac{u_{\alpha} u_{\beta}}{2 c_{s}^{4}}\left(c_{i \alpha} c_{i \beta}-c_{s}^{2} \delta_{\alpha \beta}\right)+\theta \frac{u_{\alpha} u_{\beta} u_{\gamma} c_{i \gamma}}{6 c_{s}^{6}}\left(c_{i \alpha} c_{i \beta}-3 c_{s}^{2} \delta_{\alpha \beta}\right)\right]
$$

In order to determine the weighting factors $t_{p}$, in addition to the previous constraints the isotropic $6^{\text {th }}$ order tensor of particle velocity (for two- and three-dimensional cases) must be satisfied. Therefore, the problem to eliminate the cubic nonlinear term in (2.5) becomes how to determine the weighting factors $t_{p}$ and the parameter $\theta$ in addition to the usual constraints [12-13].

The final momentum equation for D1Q5 model is,

$$
\partial_{t}(\rho u)+\partial_{x}\left(\rho u^{2}\right)=-\partial_{x} P+\partial_{x}\left[\rho(\nu+\zeta) \partial_{x} u\right]+\chi \partial_{x} \partial_{x}\left(\rho u^{3}\right)
$$

where $\chi$ is,

$$
\chi=\left(\theta \frac{\sum t_{p} c_{i}^{6}-9 c_{s}^{6}}{6 c_{s}^{6}}-1\right) \sigma
$$




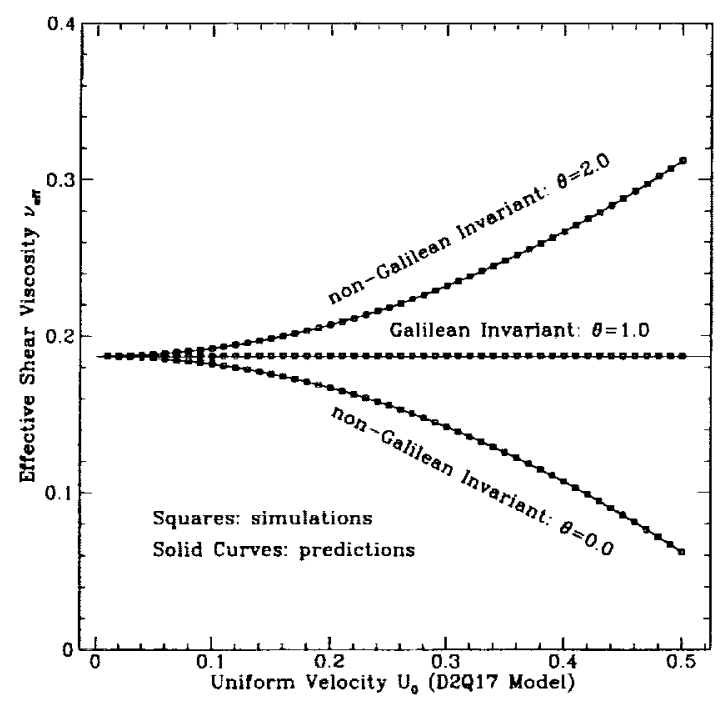

FIG. 2.1. Effective Shear viscosity $\nu$ in function of the frame-velocity $U_{0}$. The D2Q17 model with $\theta=0.0,2.0$ (nonGalilean invariant) and with $\theta=1.0$ (Galilean invariant) and $\omega=1.0$ are used. Squares are numerical results and solid curves are predictions.

$\theta=3.0$ leads to $\chi=0.0$.

For the D2Q17 model,

$$
\begin{aligned}
\partial_{t}\left(\rho u_{\alpha}\right)+\partial_{\beta}\left(\rho u_{\alpha} u_{\beta}\right)= & -\partial_{\alpha} P+\partial_{\beta}\left[\rho \nu \partial_{\beta}\left(u_{\alpha}\right)+\rho \zeta \partial_{\alpha}\left(u_{\beta}\right)\right] \\
& +\chi \partial_{\beta} \partial_{\gamma}\left(\rho u_{\alpha} u_{\beta} u_{\gamma}\right)
\end{aligned}
$$

and $\chi$ is also derived,

$$
\chi=(\theta-1) \sigma
$$

We need to replace $-\sigma$ in Eq. (2.10) with $\chi$ and $\theta=1.0$ leads to $\chi=0.0$. After some algebraic manipulations, the $c_{s}^{2}$ for the D2Q17 model is the solution of the following quadratic equation,

$$
51 X^{2}+13 X-12=0
$$

We obtain in the following table the weighting factors of these two models,

TABLE 1

Weighting factors $t_{p}$, constant $c_{s}$ and $\theta$

\begin{tabular}{||l|c|c|c|c|c|c|c||}
\hline Model & $t_{0}$ & $t_{1}$ & $t_{2}$ & $t_{4}$ & $t_{8}$ & $c_{s}^{2}$ & $\theta$ \\
\hline$D 1 Q 5$ & $1 / 2$ & $1 / 6$ & & $1 / 12$ & & 1 & 3.0 \\
\hline$D 2 Q 17$ & 0.4092905 & 0.1123018 & 0.0335591 & 0.0017273 & 0.0000891 & 0.3740845 & 1.0 \\
\hline
\end{tabular}

Using these parameters, the cubic nonlinear term is exactly cancelled out without introducing any higher order nonlinearities. Therefore, the final equations are,

$$
\begin{aligned}
\partial_{t} \rho+\partial_{\alpha}\left(\rho u_{\alpha}\right) & =0 \\
\rho\left(\partial_{t} u_{\alpha}+u_{\beta} \partial_{\beta} u_{\alpha}\right) & =-\partial_{\alpha} P+\partial_{\beta}\left[\rho \nu \partial_{\beta}\left(u_{\alpha}\right)+\rho \zeta \partial_{\alpha}\left(u_{\beta}\right)\right]
\end{aligned}
$$



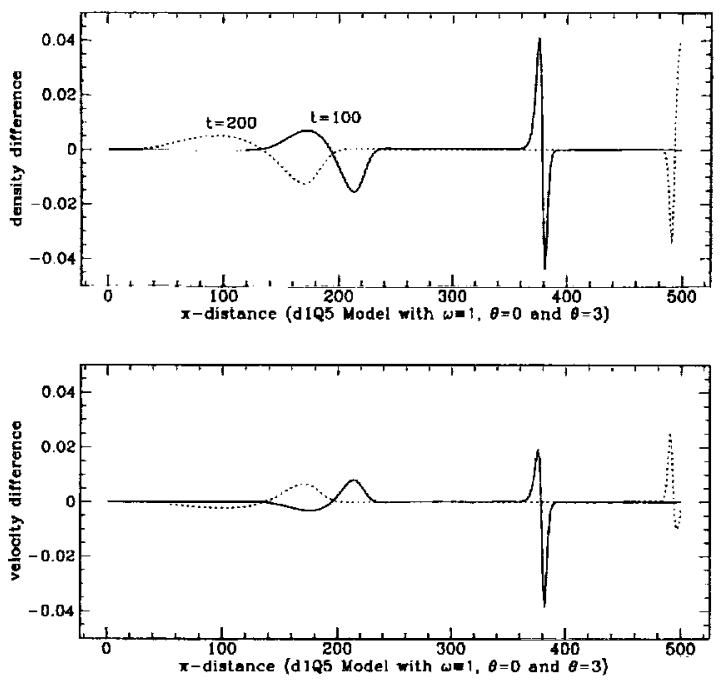

Fig. 3.1. Comparison of shock tube simulations with $(\theta=0.0)$ or without $(\theta=3.0)$ the cubic nonlinear term in the momentum equation. The D1Q5 model is used. $\omega=1.0$.

which lead to the complete Galilean invariance regardless of the velocity direction of a uniformly moving frame.

3. Simulations. Two numerical simulations have been carried out. The first one is to measure the effective shear viscosity in different moving frames. Figure 1 shows the effective shear viscosity in function of the uniform velocity $U_{0}$ with $\theta=0.0,1.0$ and 2.0. Squares are numerical simulations while solid curves are predictions based on Eq. (2.10). Only $\theta=1.0$ leads to velocity-independent viscosity which achieves the complete Galilean invariance. The neglection of this term results in relative errors of about $10 \%$ for $U_{0}=0.2$. A shock tube simulation [20] is given in Figure 2 with the following conditions: initially, left half-tube density is 3.0 while right-half density 1.0 , velocity is zero everywhere; at the two ends, bouncing-back conditions are imposed. The D1Q5 model is used to compare the cubic nonlinear effect with $\theta=0.0$ and $\theta=3.0$. The density and velocity differences are obtained at two different time steps $t=100$ and $t=200$. About $10 \%$ of difference is found due to the non-Galilean invariance. We do believe that non-Galilean invariant effect may be more significant in convection of passive scalars as discussed in the paper [9].

4. Conclusion. In this letter, we note that the current popular lattice-based hydrodynamics models are only Galilean invariant in the advection terms. The cubic nonlinear deviation term, which is a direct result of the chosen equilibrium distribution, does not satisfy the Galilean invariance. This fundamental property in required for all physical system. We develop a one-dimensional 5-velocity model $(\theta=3.0)$ and a two-dimensional 17-velocity model $(\theta=1.0)$ that restore the Galilean invariance by eliminating the cubic term. Therefore, the original Navier-Stokes equation is recovered with complete Galilean invariance. A three-dimensional model with complete Galilean invariance is under investigation and a lengthy paper of the details and applications of the present models will be published in the future [21]. An important by-product of this model is the elimination of the staggered spurious invariants [19]. For cases in which the continuum limits (long wavelength and long time) break down, effects such as rarefied gas effect [22] and finite lattice size effect [23] occur. 
5. Acknowledgments. The authors are grateful to Drs. S.Y. Chen, D. d'Humières, S. Succi and H.D. Chen for illuminating conversations.

\section{REFERENCES}

[1] U. Frisch, B. Hasslacher, and Y. Pomeau, Phys. Rev. Lett. 56, 1986, p. 1505.

[2] D. D'Humières, P. Lallemand, AND U. Frisch, Europhys. Lett. 2, 1986, p. 291.

[3] Lattice Gas Methods for PDE's, G. Doolen ed., PhysicaD 47, 1991, pp. 1-2.

[4] R. Benzi, S. Succi and M. Vergassola, Phys. Reports 222, No. 3, 1992, p. 147.

[5] D.H. Rothman and S. Zaleski, Rev. Mod. Phys. 66, 1994, p. 1417.

[6] J. Stat. Phys., Special Issue on Lattice Models, J. Lebowitz, S. Orszag and Y. Qian, eds., 81, 1995, pp. 1-2.

[7] Y.H. Qian, S. Succi And S. Orszag, Ann. Rev. Comp. Phys. III, 1995, p. 195.

[8] S.Y. Chen And G. Doolen, Ann. Rev. Fluid Mech., 1998.

[9] D. D'Humières, P. Lallemand, and G. Searby, Complex Systems 1, 1987, p. 633.

[10] G.R. McNamara and G. Zanetti, Phys. Rev. Lett. 61, 1988, p. 2332.

[11] F.J. Higuera ANd J. JimenEz, Europhys. Lett. 9, No. 7, 1989, pp. 663-668.

[12] Y.H. QIAN, Lattice Gas and Lattice Kinetic Theory Applied to the Navier-Stokes Equation, PhD thesis, University of Paris 6, 1990.

[13] Y.H. Qian, D. D'Humières, and P. Lallemand, Europhys. Lett. 17, No. 6, 1992, pp. 479-484.

[14] S.Y. Chen, H.D. Chen, D.O. Martinez, and W. Matthaeus, Phys. Rev. Lett. 67, 1991, p. 3776.

[15] H.D. Chen, S.Y. Chen, And W. Matthaeus, Phys. Rev. A 45, 1992, p. R5339.

[16] Y.H. QIAN AND S. OrSZAG, Europhys. Lett. 21, No. 3, 1993, pp. 255-259.

[17] Y. Chen, H. Ohashi, and M. Akтyama, Phys. Rev. E 50, 1994, p. 2776.

[18] Y.H. QIan AND Y. Zhou, submitted to Phys. Rev. E, 1998.

[19] Y.H. QIAN, S. OrszaG, D.H. Feng, AND M. Vallières, submitted to Phys. Rev. Lett., 1997.

[20] Y.H. QIAN, D. D'Humières, AND P. Lallemand, in Advances in Kinetic Theory and Continuum Mechanics, R. Gatignol and Soubbaramayer, eds., Springer-Verlag, 1991, p. 127.

[21] Y.H. QIAN AND Y. ZHOU, in preparation for the Phys. Fluids, 1998.

[22] Y.H. QIAN AND S.A. Orszag, J. Stat. Phys. 81, No. 1/2, 1995, p. 237.

[23] Y.H. QIAN AND S.Y. Chen, Int. J. Mod. Phys. C 8, No. 4, 1997, p. 763. 


\begin{tabular}{|c|c|c|c|}
\hline \multicolumn{3}{|c|}{ REPORT DOCUMENTATION PAGE } & $\begin{array}{l}\text { Form Approved } \\
\text { OMB No. 0704-0188 }\end{array}$ \\
\hline \multicolumn{4}{|c|}{$\begin{array}{l}\text { Public reporting burden for this collection of information is estimated to average } 1 \text { hour per response, including the time for reviewing instructions, searching existing data sources } \\
\text { gathering and maintaining the data needed, and completing and reviewing the collection of information. Send comments regarding this burden estimate or any other aspect of this } \\
\text { collection of information, including suggestions for reducing this burden, to Washington Headquarters Services, Directorate for Information Opperations and Reports, } 1215 \text { Jefferson } \\
\text { Davis Highway, Suite } 1204 \text {, Arlington, VA 22202-4302, and to the Office of Management and Budget, Paperwork Reduction Project (07040188). Washington, DC 20503. }\end{array}$} \\
\hline 1. AGENCY USE ONLY(Leave blank) & $\begin{array}{l}\text { 2. REPORT DATE } \\
\text { August } 1998\end{array}$ & \multicolumn{2}{|c|}{$\begin{array}{l}\text { 3. REPORT TYPE AND DATES COVERED } \\
\text { Contractor Report }\end{array}$} \\
\hline \multicolumn{3}{|c|}{$\begin{array}{l}\text { 4. TITLE AND SUBTITLE } \\
\text { Complete Galilean-invariant lattice BGK models for the Navier- } \\
\text { Stokes equation }\end{array}$} & \multirow[t]{2}{*}{$\begin{array}{l}\text { 5. FUNDING NUMBERS } \\
\text { C NAS1-97046 } \\
\text { WU 505-90-52-01 }\end{array}$} \\
\hline \multicolumn{3}{|l|}{$\begin{array}{l}\text { 6. AUTHOR(S) } \\
\text { Yue-Hong Qian } \\
\text { Ye Zhou }\end{array}$} & \\
\hline \multicolumn{3}{|c|}{$\begin{array}{l}\text { 7. PERFORMING ORGANIZATION NAME(S) AND ADDRESS(ES) } \\
\text { Institute for Computer Applications in Science and Engineering } \\
\text { Mail Stop 403, NASA Langley Research Center } \\
\text { Hampton, VA 23681-2199 }\end{array}$} & $\begin{array}{l}\text { 8. PERFORMING ORGANIZATION } \\
\text { REPORT NUMBER } \\
\text { ICASE Report No. } 98-38\end{array}$ \\
\hline \multicolumn{3}{|c|}{$\begin{array}{l}\text { 9. SPONSORING/MONITORING AGENCY NAME(S) AND ADDRESS(ES) } \\
\text { National Aeronautics and Space Administration } \\
\text { Langley Research Center } \\
\text { Hampton, VA 23681-2199 }\end{array}$} & $\begin{array}{l}\text { 10. SPONSORING/MONITORING } \\
\text { AGENCY REPORT NUMBER } \\
\text { NASA/CR-1998-208701 } \\
\text { ICASE Report No. } 98-38\end{array}$ \\
\hline \multicolumn{4}{|c|}{$\begin{array}{l}\text { 11. SUPPLEMENTARY NOTES } \\
\text { Langley Technical Monitor: Dennis M. Bushnell } \\
\text { Final Report } \\
\text { To appear in Europhysics Letters }\end{array}$} \\
\hline \multicolumn{2}{|c|}{$\begin{array}{l}\text { 12a. DISTRIBUTION/AVAILABILITY STATEMENT } \\
\text { Unclassified-Unlimited } \\
\text { Subject Category } 34 \\
\text { Distribution: Nonstandard } \\
\text { Availability: NASA-CASI (301)621-0390 }\end{array}$} & & 12b. DISTRIBUTION CODE \\
\hline \multicolumn{4}{|c|}{$\begin{array}{l}\text { 13. ABSTRACT (Maximum } 200 \text { words) } \\
\text { Galilean invariance has been an important issue in lattice-based hydrodynamics models. Previous models concen- } \\
\text { trated on the nonlinear advection term. In this paper, we take into account the nonlinear response effect in a } \\
\text { systematic way. Using the Chapman-Enskog expansion up to second order, complete Galilean invariant lattice BGK } \\
\text { models in one dimension }(\theta=3) \text { and two dimensions }(\theta=1) \text { for the Navier-Stokes equation have been obtained. }\end{array}$} \\
\hline \multirow{2}{*}{\multicolumn{3}{|c|}{$\begin{array}{l}\text { 14. SUBJECT TERMS } \\
\text { Boltzmann equation; lattice-based hydrodynamics models; Navier-Stokes equation; } \\
\text { Galilean invariance }\end{array}$}} & $\begin{array}{l}\text { 15. NUMBER OF PAGES } \\
11\end{array}$ \\
\hline & & & $\begin{array}{r}\text { 16. PRICE CODE } \\
\text { A03 }\end{array}$ \\
\hline $\begin{array}{l}\text { 17. SECURITY CLASSIFICATION } \\
\text { OF REPORT } \\
\text { Unclassified }\end{array}$ & $\begin{array}{l}\text { 18. SECURITY CLASSIFICATION } \\
\text { OF THIS PAGE } \\
\text { Unclassified }\end{array}$ & $\begin{array}{l}\text { 19. SECURITY CLASSIFICATION } \\
\text { OF ABSTRACT }\end{array}$ & $\begin{array}{l}\text { 20. LIMITATION } \\
\text { OF ABSTRACT }\end{array}$ \\
\hline NSN 7540-01-280-5500 & & & $\begin{array}{l}\text { Standard Form 298(Rev. 2-89) } \\
\text { Prescribed by ANSI Std. Z39-18 } \\
\text { 298-102 }\end{array}$ \\
\hline
\end{tabular}




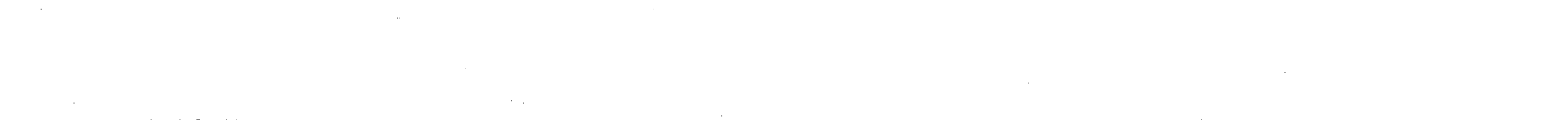

$-7$ 